\title{
Experience with invasive Candida infections
}

\author{
Hanasoge Girishkumar, Arshad M Yousuf, Jayant Chivate, Edward Geisler
}

\begin{abstract}
Summary
Between January and July 1995, 227 patients at the Bronx-Lebanon Hospital Center had positive fungal cultures. Candida spp were the most common fungi isolated. Forty-three patients with invasive disease, as indicated by fungus-positive blood cultures, became the focus of our study. $C$ albicans caused fungaemia in 21 patients $(49 \%)$. Twenty-eight patients $(65 \%)$ were less than 50 years of age; three were neonates. The most common presenting symptoms were fever, chills, and weakness (20 patients, 47\%). Thirty patients died, giving a mortality rate of $70 \%$. The patients who died stayed in the hospital an average of 49 days.

The highest mortality occurred among patients who became bacteraemic before or at the same time they became candidaemic ( 24 of 26 patients) or who were receiving broad-spectrum antibiotics (20 of 26 patients). We also found high mortality rates from invasive fungal infection among patients with HIV infection, a central venous catheter, and liver, renal, or respiratory failure. We did not find any increase in the incidence of invasive fungal infection or mortality among leukopenic or diabetic patients.
\end{abstract}

Keywords: Candida; invasive infection; risk factors

Candida spp, the major pathogenic fungi, incite disease in hosts whose local or systemic immune system is deficient, damaged, or congenitally dysfunctional. ${ }^{1}$ Those most susceptible to the development of systemic candidiasis are patients with haematologic malignancies, post-operative patients, or individuals whose immune systems have been compromised by disease or therapy. The primary source of disseminated candidiasis in patients with an adequate immune system is likely to be an infected intravenous catheter or contaminated infusion, especially hyperalimentation fluid.

According to the US National Nosocomial Infections Surveillance System of the Centers for Disease Control, the incidence of Candida as a nosocomial pathogen is increasing steadily. ${ }^{2}$ Indeed, Candida spp are the fifth most common organism isolated from blood and the seventh most common to cause nosocomial infections. ${ }^{2}$ The morbidity associated with such infection also has increased, lengthening patients' stays in hospitals and intensive care units by as much as 30 days. Among those with such infection, mortality rates are as high as $80 \% .^{3-5}$
The gastrointestinal tract is believed to be the major colonising habitat of Candida spp, especially in patients receiving broad-spectrum antibiotics. Yeasts have been isolated from the jejunum, ileum, and colon of $21-100 \%$ of hospitalised patients. ${ }^{6}$ The female genital tract and the urine of patients with indwelling Foley catheters also are frequently colonised by Candida spp. ${ }^{7}$

\section{Materials and methods}

Through a review of laboratory records, we identified 227 patients at the Bronx-Lebanon Hospital Center who had positive fungal cultures between January and July 1995. These included 110 patients with positive Candida cultures grown from bronchial aspiration lavages. Forty-three patients had funguspositive blood cultures, indicating invasive disease. These patients with invasive disease became the focus of our study.

Patients' hospital records were reviewed to obtain demographic information such as age, sex, or race. Patients' clinical courses and medical histories were recorded, as was the presence of immunosuppression from human immunodeficiency virus (HIV) infection, steroid use, or diabetes mellitus. We also documented prior or concomitant bacterial septicaemia and administration of antibiotics, chemotherapy, and parenteral alimentation, as well as risk factors such as central venous catheterization, mechanical ventilation, and admission to an intensive care unit. Fungaemia was considered to be nosocomial if there was no evidence of fungal infection at the time of admission but blood cultures became positive more than 72 hours after admission.

The diagnosis of Candida infection relied on direct inspection of freshly obtained specimens for the presence of organisms microscopically consistent with Candida spp and the recovery of Candida spp in cultures of blood, body fluids, or tissues. Direct microscopic examination of specimens not only provided semiquantitative estimates of the amount of Candida spp, but also supplemented culture results. Cultures were obtained using a standard broth blood culture system (Bactec-Johnston Laboratories, Towson, MD).

Intravascular catheters are an important potential source of fungaemia. When such a catheter was removed from a patient, a $5-\mathrm{cm}$ portion of its distal segment was sent to the laboratory. The catheter segment was rolled over the surface of a blood agar plate, and cultured in a tube of broth medium. The plate was 
incubated for 48 hours and colonies were counted.

\section{Results}

PATIENT DEMOGRAPHICS

The study population consisted of 17 women $(40 \%)$ and 26 men (61\%). Thirty (70\%) were African-Americans and $13(30 \%)$ were of Hispanic origin. Three patients $(7 \%)$ were from the neonatal intensive care unit. Twenty-eight patients $(65 \%)$ were less than 50 years of age (age range, 16 days to 86 years); the mean age was 50 years.

SYMPTOMATOLOGY AND CLINICAL COURSE

The most common presenting symptoms were fever, chills, and weakness (20 patients, 47\%). End-organ involvement such as pneumonia occurred in 11 patients $(26 \%)$. All patients had a routine ophthalmologic examination and six patients $(14 \%)$ were found to have Candida endophthalmitis.

The blood of 33 patients (77\%) grew various species of Candida. Specifically, $C$ albicans caused fungaemia in 21 patients $(49 \%), C$ glabrata was identified in five patients $(12 \%), C$ parapsilosis in four patients ( $9 \%$ ), and $C$ tropicalis in three patients $(7 \%)$. Other causative organisms were Cryptococcus neoformans (seven patients, 16\%), Aspergillus spp (two patients, 5\%), and Penicillium spp in one patient. Two patients' blood and cerebrospinal fluid grew Cryptococcus neoformans. C glabrata was grown from both the blood and bronchial aspiration lavage of one patient. Another patient's blood grew $C$ glabrata, whereas his bronchial aspiration lavage grew $C$ albicans. $C$ tropicalis was grown from the blood, but $C$ albicans from the bronchial aspiration lavage of two patients. In 10 cases $(23 \%)$, yeast was grown from blood after the patients had died.

RISK FACTORS AND UNDERLYING ILLNESS

Twenty-six patients $(61 \%)$ had been bacteraemic before becoming candidaemic. The underlying infections included Staphylococcus epidermidis sepsis (six patients, 14\%), Staphylococcus aureus (six patients, 14\%), Enterococcus spp (two patients, 5\%), Salmonella spp, (two patients, 5\%) and Klebsiella spp (two patients, $5 \%$ each), and Enterobacter, (one patient). Seven patients had systemic inflammatory response syndrome but no organism was grown from their blood. Sixteen patients $(37 \%)$ had leukopenia (white cell count of less than $4 \times$ $10 \% / \mathrm{ml})$. Thirteen patients $(30 \%)$ had diabetes mellitus, while 27 (63\%) had altered liver function, (bilirubin and transaminase levels that were persistently greater than twice the normal value), and 24 (56\%) had respiratory failure (needed ventilatory support). Six patients $(14 \%)$ underwent surgery, two for bowel obstruction resulting from carcinoma of the colon. The other surgical procedures, each done on one patient, were total hip replacement, exploratory laparotomy for caecal perforation, drainage of a pancreatic pseudocyst, and perforated appendix. Twenty-two patients $(51 \%)$ had HIV infection. Two patients $(5 \%)$ reported alcohol and cocaine abuse, but their immune status was not known. The mother of one neonatal patient was a cocaine abuser.

TREATMENT AND MORTALITY

The 30 patients $(70 \%)$ who died had an average hospital length of stay of 49 days. Of the 24 patients (56\%) who received amphotericin $\mathrm{B}$ alone, 14 died. Of the four patients (9\%) who received fluconazole alone, all died. Eleven patients received both fluconazole and amphotericin B.

\section{Discussion}

Nolla-Salas et $a l^{8}$ found that patients who received antifungal therapy no later than 48 hours after the onset of candidaemia had a higher probability of survival compared with patients who did not receive antifungal therapy until at least 48 hours after the onset of candidaemia. Detection of Candida spp in blood culture depends on the volume of the blood samples, the condition of the medium and atmosphere, the concentrations of Candida spp within the bloodstream, and the species of Candida present. $C$ albicans, $C$ tropicalis, and $C$ parapsilosis tend to be detected 1-3 days earlier than $C$ krusei and $C$ glabrata, which can require longer times for growth. ${ }^{9}$ Many early attempts to diagnose invasive candidiasis by means other than blood cultures failed. However, recent advances in antigen purification, monoclonal antibody production, recombinant DNA techniques, and polymerase chain reaction have made such diagnosis possible. Among the potential markers of invasive candidiasis are Candida cell-wall antigens such as mannans; Candida metabolites, particularly darabinitol; and Candida cytoplasmic antigen (Candida enolase and Candida heat shock protein-HSP 90). ${ }^{9}$ Although these tests are most useful for early diagnosis and monitoring the response of invasive candidiasis to therapy, they cannot replace microbiological culture in diagnosis of this infection. Also, these tests are not universally available.

All patients in our study had positive fungal blood culture, and $70 \%$ died in spite of treatment. The mortality among patients with this infection is high, ranging from 60 to $70 \% .{ }^{8-10}$ Several studies have indicated risk factors for fungaemia. ${ }^{810-12}$ Early treatment of patients with these risk factors confers a higher probability of survival than does delayed therapy. ${ }^{8}$

The results of prophylactic administration of systemic antifungal agents to persistently febrile patients receiving antibiotic therapy has been extensively studied. A study by the European Organisation for Research and Treatment of Cancer examined the empirical use of amphotericin B for leukopenic patients who remained febrile for 4 days despite the administration of antibiotics. ${ }^{13}$ It was found that amphotericin B was beneficial in resolving these patients' fever.

According to Walsh et al, ${ }^{14}$ leukopenia becomes a major risk factor for invasive fungal infection only if it lasts longer than 3 weeks, is 
profound (less than $100 \times 10^{9} / 1$ blood), and occurs secondary to cytotoxic chemotherapy or ablation radiation therapy. We found no increase in mortality among leukopenic patients who developed invasive fungal infection as compared to patients with a normal white cell count.

As noted by Eubanks et $a l,{ }^{15}$ we also did not find a higher mortality rate in patients with diabetes mellitus who developed invasive fungal infection. However, our patients with HIV infection had higher mortality from invasive fungal infection than those who were HIVseronegative. We also found increased mortality among patients who were bacteraemic before or at the same time they became candidaemic, or who were receiving broad-spectrum antibiotics. The predominance of Candida spp over normal microbial flora has long been noted after the administration of oral or parenteral antibacterial agents that eliminate bacterial competition. ${ }^{5}$

Central venous catheters are a major cause of candidaemia. We noted increased mortality from invasive fungal infection in patients with central venous catheters compared to patients who did not require a central venous line or other long-term tubes or catheters. The question of whether the catheter infects the patient or the patient infects the catheter so that it becomes the secondary nidus for septicaemia remains unresolved. Karabinis et $a l^{16}$ found that central venous catheterisation

1 Rinaldi MG. Biology and pathogenicity of Candidal species. n: Bodey GP, ed. Candidiasis: pathogenesis, diagnosis and treatment, 2nd edn. New York: Raven Press; 1993; pp 1-20.

2 Banerjee SN, Emori TG, Culver DH, et al. Secular trends in nosocomial primary bloodstream infections in the United States, 1980-1989. Am $\mathcal{F}$ Med 1989;91:86-9.

3 States, 1980-1989. Am f Med 1989;91:86-9. patients. Am f Surg 1996;71:374-82.

4 Beck-Sague CM, Jarvis WR. Secular trends in the epidemiBeck-Sague CM, Jarvis WR. Secular trends in the epidemiology of nosocomial fungal infections in the
1980-1990. F Infect Dis 1993;167:1247-51.

5 Wey SB, Mori M, Pfaller MA. Risk factors for hospitalacquired candidemia: a matched case- control study. Arch Intern Med 1989;149:2349-53.

6 Odds FC. Candida infections: an overview. Crit Rev Microbiol 1987;15:1-5.

7 Wade JC. Epidemiology of Candida infections. In: Bodey GP, ed. Candidiasis: pathogenesis, diagnosis and treatment, 2nd edn. New York: Raven Press; 1993; pp 85-107.

8 Nolia-Salas J, Sitges-Serra A, Leon-Gill C. Candidemia in non-neutropenic critically ill patients: analysis of prognostic factors and assessment of systemic antifungal therapy. Study group of fungal infection in the ICU. Intens Care Med 1997; 23:23-30.

9 Walsh TJ, Pizzo PA. Laboratory diagnosis of candidiasis. In:Bodey GP, ed. Candidiasis: pathogenesis, diagnosis and In:Bodey GP, ed. Candidiasis: pathogenesis, diagnosis and
treatment, 2nd edn. New York: Raven Press; 1993; treatment,

10 Voss A, Le Noble JL, Verduyn-Lunel FM, Meis JF. Candidemia in intensive care unit patients: risk factors for mortality. Infection 1997;25:8-11. was an important risk factor for candidaemia in patients with cancer.

In our study, other factors leading to increased mortality from invasive fungal infection included renal failure, hepatic failure, and respiratory failure. Multiple organ failure in these patients could reflect lethal dissemination of candidiasis. The liver, kidneys, and lungs are among the organs most commonly involved in disseminated candidiasis. ${ }^{17}$ It is possible that those failing organs provide sequestered areas where Candida spp can proliferate and avoid the immune response reactions. ${ }^{15}$

Anti-fungal prophylaxis appears to be an attractive alternative for high-risk patients. The development of resistance to therapy, and the possible side-effects of this treatment, should be assessed before treatment of any patient is contemplated. Al Soub and Estinoso ${ }^{18}$ found no significant difference between patients treated with fluconazole and amphotericin B. Further studies with large numbers of patients are needed to assess the effect of early antifungal therapy on the mortality associated with candidaemia and to determine the appropriate medication, dose, and duration of treatment. As fluconazole has gained widespread use, the emergence of fluconazole-resistant Candida spp has been noted, especially in HIV-infected patients. The lack of in vitro activity of fluconazole against $C$ krusei supports this clinical finding. ${ }^{19}$

11 Gonzalez CE, Venzon D, Lee S, Mueller BU, Pizzo PA, Walsh TJ. Risk factors for fungemia in children infected with human immunodeficiency virus- a case control study. Clin Infect Dis 1996;23:515-21.

12 Krcmery V, Oravcova E, Spanik S, Mrazova-Studena M, Trupl J, Kunova A. Nosocomial breakthrough fungemia during antifungal prophylaxis or empirical antifungal therapy in 41 cancer patients receiving antineoplastic chemotherapy: analysis of etiology, risk factors and outcome. F Antimicrob Chem 1998;41:373-80.

13 EORTC International Antimicrobial Therapy Cooperative Group. Empiric antifungal therapy in fibrile granulocytopenic patients. Am f Med 1989;86:668-72.

14 Walsh TJ, Lee JW. Prevention of invasive fungal infections in patients with neoplastic diseases. Clin Infect Dis 1993; 17(S2):S468-S480.

15 Eubanks PJ, Virgilio CD, Klein S, Bongard F. Candida sepsis in surgical patients. Am F Surg 1993;166:617-20.

16 Karabinis A, Hill C, Leclercq B, et al. Risk factors for candidemia in cancer patients: a case control study. $\mathcal{F}$ Clin Microbiol 1988;26:429-32.

17 Meunier F. Candidiasis. Eur F Clin Microbiol Infect Dis 1989; 8:438-47.

18 Al Soub H, Estinoso W. Hospital acquired candidemia: experience from a developing country. $\mathcal{F}$ Hosp Infect 1997;35:141-7

19 Wingard JR, Merz WG, Rinaldi MG, et al. Increase in Candida krusei infection among patients with bone marrow transplantation and neutropenia treated prophylactically with fluconazole. N Engl f Med 1991;25:1274-7. 